\title{
Distribution of the ratio of an unordered eigenvalue to the trace of a complex central Wishart matrix and its application to cooperative spectrum sensing
}

\author{
Dongming Wang ${ }^{a}$, Hao Wei, Yufei Wu, and Ruixun Liu \\ National Mobile Communications Research Lab., Southeast University, \\ Nanjing, Jiangsu, 210096, China \\ a)wangdm@seu.edu.cn
}

\begin{abstract}
In this paper, we derive the closed-form expressions of the probability distribution function (PDF) and cumulative distribution function (CDF) of the ratio of an unordered eigenvalue to the trace of a complex central Wishart matrix. Using the random matrix result, we study the eigenvalue-based spectrum sensing algorithm for cognitive ratio. We apply the goodness of fit test to the ratio of an unordered eigenvalue to the trace of the sample covariance matrix. Finally, we compare the performances between the proposed algorithm and the well-known eigenvalue-based spectrum sensing algorithms in cooperative systems.
\end{abstract}

Keywords: Wishart matrix, spectrum sensing

Classification: Wireless Communication Technologies

\section{References}

[1] A. M. Tulino and S. Verdú, "Random matrix theory and wireless communications," Foundations and Trends in Communications and Information Theory, vol. 1, no. 1, pp. 1-182, 2004. DOI:10.1561/0100000001

[2] Y. Zeng and Y. C. Liang, "Eigenvalue-based spectrum sensing algorithms for cognitive radio," IEEE Trans. Commun., vol. 57, no. 6, pp. 1784-1793, Jun. 2009. DOI:10.1109/TCOMM.2009.06.070402

[3] Y. Zeng, Y. C. Liang, and R. Zhang, "Blindly combined energy detection for spectrum sensing in cognitive radio," IEEE Signal Process. Lett., vol. 15, no. 10, pp. 649-652, Oct. 2008. DOI:10.1109/LSP.2008.2002711

[4] P. Wang, J. Fang, N. Han, and H. Li, "Multiantenna-assisted spectrum sensing for cognitive radio," IEEE Trans. Veh. Technol., vol. 59, no. 4, pp. 1791-1800, May 2010. DOI:10.1109/TVT.2009.2037912

[5] L. Wei, O. Tirkkonen, P. Dharmawansa, and M. R. McKay, "On the exact distribution of the scaled largest eigenvalue," Proc. IEEE International Conference on Communications (ICC12), Ottawa, Canada, Jun. 2012.

[6] H. Wang, E. Yang, Z. Zhao, and W. Zhang, "Spectrum sensing in cognitive radio using goodness of fit testing," IEEE Trans. Wireless Commun., vol. 8, no. 11, pp. 5427-5430, Nov. 2009. DOI:10.1109/TWC.2009.081586 
[7] Y. Lin, C. He, L. Jiang, and D. He, "Cooperative spectrum sensing based on stochastic resonance in cognitive radio networks," Sci. China Inf. Sci., vol. 57, no. 2, pp. 1-10, 2014. DOI:10.1007/s11432-013-5010-7

[8] H. Shin and J. H. Lee, "Capacity of multiple-antenna fading channels: spatial fading correlation, double scattering, and keyhole," IEEE Trans. Inf. Theory, vol. 49, no. 10, pp. 2636-2647, Oct. 2003. DOI:10.1109/TIT.2003.817439

[9] O. Besson and L. L. Scharf, "CFAR matched direction detector," IEEE Trans. Signal Process., vol. 54, no. 7, pp. 2840-2844, Jul. 2006. DOI:10.1109/TSP. 2006.874782

[10] I. S. Gradshteyn and I. M. Ryzhik, Table of Integrals, Series, and Products, 7th ed., Academic, New York, 2007.

\section{Introduction}

Wishart matrices which have been known for nearly a century are of great importance in multivariate statistical theory. In the last decade, the eigenvalue statistics of complex Wishart matrices have found various applications in the wireless communications systems [1].

Most recently, due to the emergence of cognitive radio, the study on the eigenvalue ratio of complex central Wishart matrices has been attracting much attention. In [2], the eigenvalue-based spectrum sensing was firstly proposed for cognitive radio systems. For the blind eigenvalue-based spectrum sensing, the eigenvalue ratio plays an important role. In [2], maximum-minimum eigenvalue (MME) detection was proposed, where the test statistic is the ratio of the maximum eigenvalue to the minimum eigenvalue. In $[3,4]$, the blind generalized likelihood ratio test (B-GLRT) was proposed, where the test statistic is the ratio of the maximum eigenvalue to the sum of the eigenvalues. Using the random matrix theory, some of the researchers have studied the performance of the eigenvaluebased spectrum sensing. In [5], the statistics properties of the eigenvalue ratios were studied, and the closed-form expressions for the probability distribution function (PDF) and cumulative distribution function (CDF) of the eigenvalue ratios were derived.

In this paper, we study the spectrum sensing by using the new result of random matrix theory. Firstly, we derive the closed-form expressions of the PDF and CDF of the ratio of an unordered eigenvalue to the trace of a complex central Wishart matrix. To the best of the authors' knowledge, this is the first time to get those closed-form expressions. Using the random matrix results, we apply the goodness of fit test [6] for the ratio of an unordered eigenvalue to the trace of the sample covariance matrix. The performance of the spectrum sensing algorithm is evaluated for cooperative spectrum sensing [7]. Simulation results demonstrate that under the scenario of multiple cognitive users and multiple primary users, compared with MME and B-GLRT, the proposed algorithm has good performance.

\section{PDF and CDF of a normalized unordered eigenvalue of complex central Wishart matrix}

Since the trace of a complex Wishart matrix is equal to the sum of all the 
eigenvalues, in the following part, the ratio of an unordered eigenvalue to the trace of a complex central Wishart matrix is also called a normalized unordered eigenvalue of a complex central Wishart matrix.

Theorem 1. Let $\mathbf{H}$ be a complex Gaussian $(N \times K)$ random matrix with circularly symmetric zero-mean, unit variance, i.i.d. entries. Assume that $N \leq K$. Let $\lambda$ be an unordered non-zero eigenvalue of $\mathbf{H H}^{\mathrm{H}}$. A normalized unordered eigenvalue of $\mathbf{H H}^{\mathrm{H}}$ is defined as

$$
\tilde{\lambda}=\frac{\lambda}{\operatorname{Tr}\left(\mathbf{H H}^{\mathrm{H}}\right)}, \quad 0<\tilde{\lambda} \leq 1 .
$$

The PDF of $\tilde{\lambda}$ is given by

$$
\begin{aligned}
f_{\tilde{\lambda}}(x)= & \frac{(K N-1) !}{N} \sum_{i=0}^{N-1} \sum_{j=0}^{i} \sum_{l=0}^{2 j}\left[\frac{(-1)^{l}(2 j) !}{2^{2 i-l} j ! l !(K-N+j) !}\right. \\
& \cdot\left(\begin{array}{c}
2 i-2 j \\
i-j
\end{array}\right)\left(\begin{array}{c}
2 j+2 K-2 N \\
2 j-l
\end{array}\right) \\
& \left.\cdot \frac{x^{K-N+l}(1-x)^{K N-K+N-l-2}}{(K N-K+N-l-2) !}\right] .
\end{aligned}
$$

\section{Proof: See A.}

By using the Binomial expansion, the CDF of $\tilde{\lambda}$ is given by

$$
\begin{aligned}
F_{\tilde{\lambda}}(x)= & \frac{(K N-1) !}{N} \sum_{i=0}^{N-1} \sum_{j=0}^{i} \sum_{l=0}^{2 j} \sum_{n=0}^{K N-K+N-l-2} \\
& {\left[\begin{array}{c}
(-1)^{l+n}(2 j) !\left(\begin{array}{c}
2 i-2 j \\
i-j
\end{array}\right)\left(\begin{array}{c}
2 j+2 K-2 N \\
2^{2 i-l}(K-N+l+n+1) j ! l ! n !(K-N+j) !
\end{array}\right. \\
\\
\cdot \frac{x^{K-N+l+n+1}}{(K N-K+N-l-n-2) !}
\end{array}\right] }
\end{aligned}
$$

Fig. 1 shows the analytical PDF presented in Theorem 1 and the empirically generated PDF. It is demonstrated that the analytical and empirical results are in perfect agreement. Fig. 2 gives the analytical CDF and the empirically generated CDF. It can be seen that analytical expression given in (2) is consistent with the empirical data.

\section{Cooperative spectrum sensing}

In this section, we introduce the signal model of the cooperative spectrum sensing, and then we give the detailed procedure of the goodness of fit test for the normalized eigenvalues of the sample covariance matrix. 


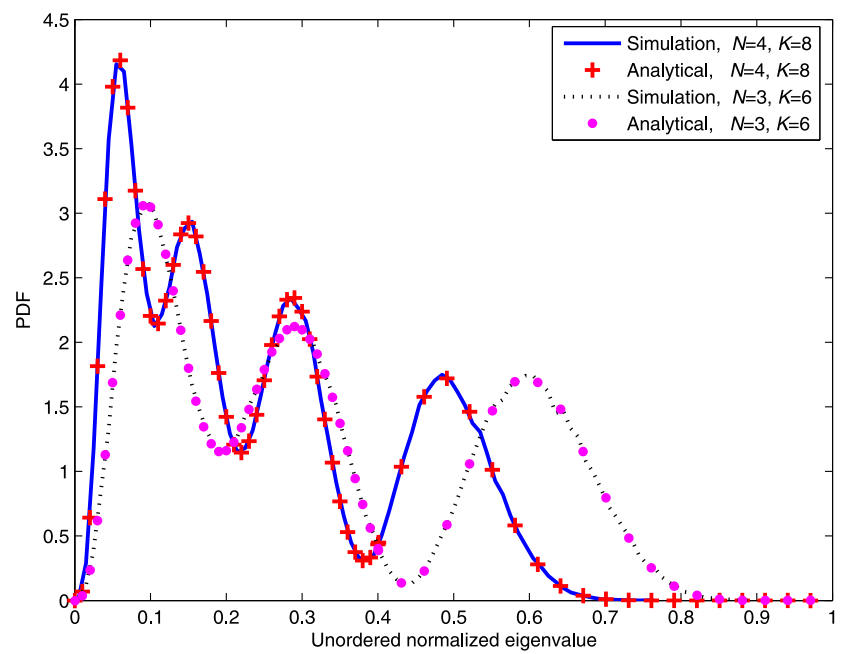

Fig. 1. The PDF of a normalized unordered eigenvalue of complex Wishart matrices.

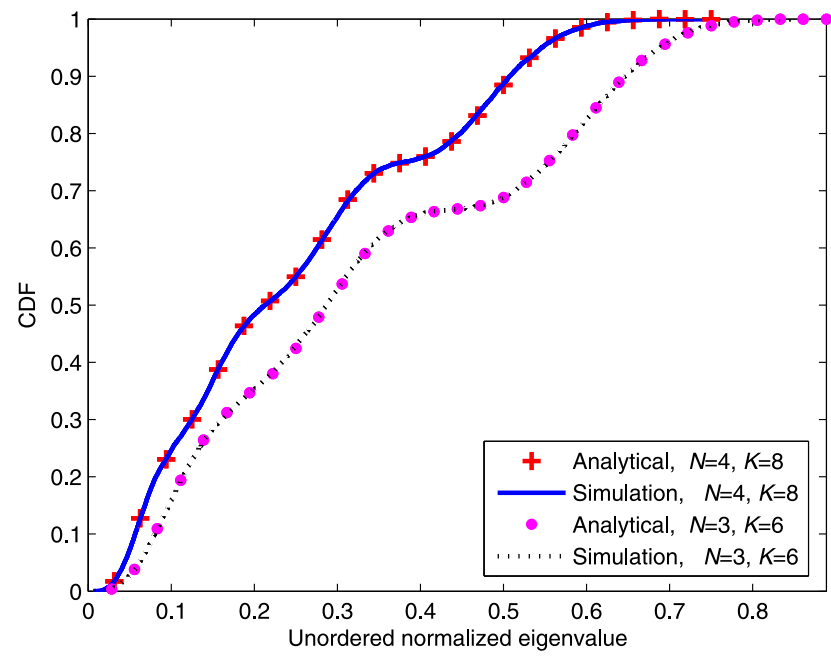

Fig. 2. The CDF of a normalized unordered eigenvalue of complex Wishart matrices.

\subsection{Signal model of cooperative spectrum sensing}

We consider a cooperative spectrum sensing problem with $N$ cognitive users and $M(N \geq M)$ primary users and each user is equipped with single antenna. Each cognitive user receives $K$ samples. Let the the hypothesis of idle channel and the hypothesis of active primary user be $H_{0}$ and $H_{1}$, respectively. The received signal at $H_{0}$ and $H_{1}$ is modeled as follows:

$$
\begin{aligned}
& H_{0}: \mathbf{y}=\mathbf{w} \\
& H_{1}: \mathbf{y}=\mathbf{G x}+\mathbf{w},
\end{aligned}
$$

where $\mathbf{y} \in \mathbb{C}^{N}$ denotes the received signals of the cognitive users, $\mathbf{G}$ denotes the $N \times M$ channel matrix, $\mathbf{x} \in \mathbb{C}^{M}$ denotes the signal of the primary users. Here we assume $\mathbf{w}$ as circularly symmetric complex Gaussian noise, i.e., $\mathbf{w} \sim \mathcal{C N}\left(\mathbf{0}, \sigma^{2} \mathbf{I}_{\mathbf{N}}\right)$. 
The sample covariance matrix of the received signal is given by

$$
\mathbf{R}=\frac{1}{K} \sum_{k=0}^{K-1} \mathbf{y}(k) \mathbf{y}^{\mathrm{H}}(k)
$$

The eigenvalues of $\mathbf{R}$ are denoted as $\lambda_{1}, \ldots, \lambda_{N}$, and the normalized eigenvalues are defined by $\tilde{\lambda}_{n}=\lambda_{n} / \operatorname{Tr}(\mathbf{R})$.

\subsection{Anderson-Darling based sensing with normalized eigenvalues}

In [6], Anderson-Darling (AD) based goodness of fit test is proposed for spectrum sensing. In this paper, to avoid the estimation of noise variance, we apply the algorithm to test the normalized eigenvalues.

The basic idea is as follows. If there is no signal transmission by the primary users, $\mathbf{R}$ is a Wishart matrix, and a normalized unordered eigenvalue $\tilde{\lambda}_{n}$ is approaching the known distribution, $F_{\tilde{\lambda}}(x)$. Otherwise, in the presence of the primary users' signal, $\tilde{\lambda}_{n}$ is different from the known distribution. Therefore, spectrum sensing can be formulated as a goodness of fit testing problem. In this paper, the goodness of fit test based on Anderson-Darling is adopted [6]. The steps are given as follows:

1. Find out the threshold $\gamma$ for a given probability of false alarm, either with simulation or with the equation [6, eq. (7)]:

2. Sort the observations $\tilde{\lambda}_{n}$ in increasing order. Assume that the sorted observations are $\tilde{\lambda}_{1}, \ldots, \tilde{\lambda}_{N}$.

3. With the known CDF in (2), calculate $z_{n}=F_{\tilde{\lambda}}\left(\tilde{\lambda}_{n}\right)$.

4. Calculate the test statistic:

$$
\tau=-\frac{1}{N} \sum_{n=1}^{N}(2 n-1)\left[\ln \left(z_{n}\right)+\ln \left(1-z_{N+1-n}\right)\right]-N
$$

5. Make a decision: if $\tau \leq \gamma$ that channel is idle, else if $\tau>\gamma$, we consider channel is busy.

\subsection{Simulation results}

In the simulations, multiple primary users and multiple cooperative receivers are used to evaluate the performance of probability of detection. Simulation results are obtained by using complex Gaussian random primary signal and independent and identically distributed (i.i.d.) complex Gaussian noise samples. The entries of the channel matrix $\mathbf{G}$ are i.i.d. circularly symmetric complex Gaussian random variable with zero-mean and unit variance.

Fig. 3 shows the performance of probability of detection of B-GLRT, MME, and AD-based sensing with the normalized eigenvalues. For $N=4, M=4$ and $K=10$, the performance of AD sensing is significantly better than that of both B-GLRT and MME. The performance gaps become small with the decreasing of $M$ or the increasing of the number of samples. But, when $M$ is larger than one, $\mathrm{AD}$ sensing usually has good performance because it explores the statistics of all the eigenvalues. 


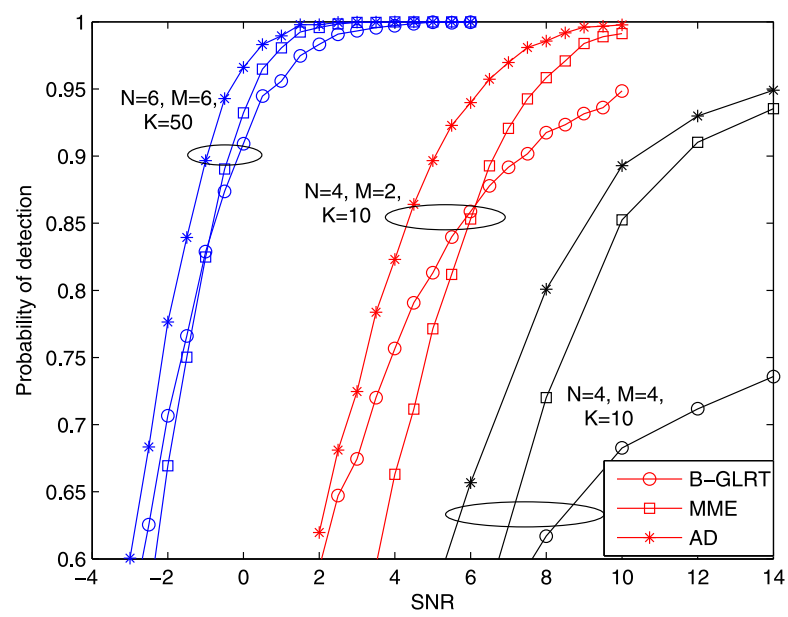

Fig. 3. Comparison of detection probability for B-GLRT, MME and AD-sensing with different SNR values.

\section{Conclusions}

In this paper, we present the closed-form expression of the PDF and CDF for a normalized unordered eigenvalue of a complex central Wishart matrix, and study its application to cooperative spectrum sensing. With the CDF, we apply the AD-based goodness of fit test for the normalized eigenvalues of the sample covariance matrix. Simulation results show that when the number of primary users is larger than one, the performance of $\mathrm{AD}$ sensing is better than that of both B-GLRT and MME.

\section{A Proofs of Theorem 1}

Let the PDFs of an unordered eigenvalue, the sum of the eigenvalues, and a normalized unordered eigenvalues be $f_{\lambda}(x), f_{\chi}(x)$, and $f_{\tilde{\lambda}}(x)$, respectively. According to [8], we have

$$
\begin{aligned}
f_{\lambda}(x)= & \frac{1}{N} \sum_{i=0}^{N-1} \sum_{j=0}^{i} \sum_{l=0}^{2 j}\left[\frac{(-1)^{l}(2 j) !}{2^{2 i-l} j ! l !(K-N+j) !}\right. \\
& \left.\cdot\left(\begin{array}{c}
2 i-2 j \\
i-j
\end{array}\right)\left(\begin{array}{c}
2 j+2 K-2 N \\
2 j-l
\end{array}\right) x^{K-N+l} e^{-x}\right] .
\end{aligned}
$$

Note that the sum of all eigenvalues of $\mathbf{H} \mathbf{H}^{\mathrm{H}}$ follows central Chi-square distribution with $2 K N$ degrees of freedom, that is

$$
f_{\chi}(x)=\frac{1}{\Gamma(K N)} x^{K N-1} e^{-x},
$$

where $\Gamma(\cdot)$ denotes the Gamma function.

As shown in [9], a normalized eigenvalue is independent of the sum of the eigenvalues. Then, according to [5], the PDF of a normalized unordered eigenvalue can be obtained by the following contour integral

$$
f_{\tilde{\lambda}}(x)=\frac{1}{2 \pi \imath} \int_{c-l \infty}^{c+l \infty} x^{-z} \frac{\mathcal{M}_{z}\left(f_{\lambda}(x)\right)}{\mathcal{M}_{z}\left(f_{\chi}(x)\right)} \mathrm{d} z
$$

where

$$
\mathcal{M}_{z}(f(x))=\int_{0}^{\infty} x^{z-1} f(x) \mathrm{d} x
$$

denotes the Mellin transform of $f(x)$. 
The Mellin transform of $f_{\lambda}(x)$ can be computed as

$$
\begin{aligned}
\mathcal{M}_{z}\left(f_{\lambda}(x)\right)= & \frac{1}{N} \sum_{i=0}^{N-1} \sum_{j=0}^{i} \sum_{l=0}^{2 j}\left[\frac{(-1)^{l}(2 j) !}{2^{2 i-l} j ! l !(K-N+j) !}\right. \\
& \cdot\left(\begin{array}{c}
2 i-2 j \\
i-j
\end{array}\right)\left(\begin{array}{c}
2 j+2 K-2 N \\
2 j-l
\end{array}\right) \\
& \cdot \Gamma(K-N+l+z)] .
\end{aligned}
$$

where

$$
\Gamma(K-N+l+z)=\int_{0}^{\infty} x^{z-1} x^{K-N+l} e^{-x} \mathrm{~d} x .
$$

The Mellin transform of $f_{\chi}(x)$ can be computed as

$$
\begin{aligned}
\mathcal{M}_{z}\left(f_{\chi}(x)\right) & =\int_{0}^{\infty} x^{z-1} \frac{1}{\Gamma(K N)} x^{K N-1} e^{-x} d x \\
& =\frac{\Gamma(z+K N-1)}{\Gamma(K N)} .
\end{aligned}
$$

Substituting (8) and (9) into (7), we obtain

$$
\begin{aligned}
f_{\tilde{\lambda}}(x)= & \frac{\Gamma(K N)}{N} \sum_{i=0}^{N-1} \sum_{j=0}^{i} \sum_{l=0}^{2 j}\left[\frac{(-1)^{l}(2 j) !}{2^{2 i-l} j ! l !(K-N+j) !}\right. \\
& \cdot\left(\begin{array}{c}
2 i-2 j \\
i-j
\end{array}\right)\left(\begin{array}{c}
2 j+2 K-2 N \\
2 j-l
\end{array}\right) \\
& \left.\cdot \frac{1}{2 \pi l} \int_{c-l \infty}^{c+l \infty} x^{-z} \frac{\Gamma(z+K-N+l)}{\Gamma(z+K N-1)} d z\right] .
\end{aligned}
$$

With definition of the Meijer's G function [10], we have

$$
\begin{aligned}
& \frac{1}{2 \pi l} \int_{c-l \infty}^{c+l \infty} x^{-z} \frac{\Gamma(z+K-N+l)}{\Gamma(z+K N-1)} d z \\
& \quad=\mathcal{G}_{1,1}^{1,0}\left(x \mid \begin{array}{c}
K N-1 \\
K-N+l
\end{array}\right)
\end{aligned}
$$

As shown in [5],

$$
\mathcal{G}_{1,1}^{1,0}\left(x \mid \begin{array}{l}
a \\
b
\end{array}\right)=\frac{x^{b}(1-x)^{a-b-1}}{(a-b-1) !} \mathcal{U}(1-x),
$$

where $\mathcal{U}(\cdot)$ denotes the Heaviside step function. Note that $0<\tilde{\lambda} \leq 1$, with (12) and (11), (10) can be simplified to (1).

\section{Acknowledgments}

This work was supported in part by the National Basic Research Program of China (973 Program 2013CB336600), the Natural Science Foundation of China (NSFC) under grants 61271205, 61501113, 61521061, 61372100, and China High-Tech 863 Program under Grant No. 2014AA01A704, the Jiangsu provincial NSF under grant BK20150630, the Hong Kong, Macao and Taiwan Science \& Technology Cooperation Program of China (Grant No. 2014DFT10290). 